\title{
Low Temperature, Low Pressure CMOS Compatible Cu-Cu Thermo-compression Bonding with Ti Passivation For 3D IC Integration
}

\author{
${ }^{1}$ Asisa Kumar Panigrahi, ${ }^{1}$ Satish Bonam, ${ }^{1}$ Tamal Ghosh, ${ }^{1}$ Siva Rama Krishna Vanjari and ${ }^{1}$ Shiv Govind Singh \\ ${ }^{1}$ Indian Institute of Technology Hyderabad \\ Department of Electrical Engineering \\ Ordnance Factory Estate, Yeddumailaram 502205 \\ Telangana, INDIA \\ Phone: +91-8332023798, Fax: (040) 23016032 \\ E-mail: ee13p1009@iith.ac.in
}

\begin{abstract}
In this paper, we report the methodology of achieving low temperature, low pressure CMOS compatible Wafer-onWafer (WoW) $\mathrm{Cu}-\mathrm{Cu}$ thermo-compression bonding using optimally chosen ultra-thin layer of Titanium (Ti) as a passivation layer. We systematically studied the effects of $\mathrm{Ti}$ thickness on bonding quality via its effects on surface roughness, oxidation prevention and inter diffusion of $\mathrm{Cu}$. Through this study, we have found that a Ti thickness of $3 \mathrm{~nm}$ not only results in excellent bonding but also leads to a reduction in operating pressure to $2.5 \mathrm{bar}$ and temperature to $175^{\circ} \mathrm{C}$. The reduction in pressure is more than an order of magnitude lower relative to the current state-of-the-art. The lower operating pressure and temperature manifest themselves in a very good homogenous bond further highlighting the efficacy of our approach. Finally, our results have been corroborated by evidence from AFM study of the $\mathrm{Cu} / \mathrm{Ti}$ surface prior to bonding. The bond strength of $\mathrm{Cu}-\mathrm{Cu}$ as measured by Instron Microtester measurement system is found to be $190 \mathrm{MPa}$ which compares very well with the reported literatures.
\end{abstract}

\section{Introduction}

Ever-growing consumer demand for smaller, faster electronics with new functionalities is a resultant of the efforts of researchers to follow Moore's Law meticulously. With the primary focus on increasing no. of transistors per unit area, the semiconductor industries pushed towards the extreme limit to make devices very small in an effort to increase the speed of operation. However, recently the focus of research has shifted towards the reduction in the delay caused by interconnects as they are becoming the bottleneck in enhancing the circuit performance. The pursuit for short interconnects has led to the concept of 3D integration wherein several thin IC chips are interconnected using vertical bonding [1]. The major advantage of 3D IC integration is that it allows heterogeneous integration of high end technologies like Logic, memory, RF etc. 3D integration offers a way for higher density, higher performance, higher functionality, and smaller form factor. 3D IC integration is typically achieved using various stacking options like Wafer-on-Wafer (WoW), Chip-on-Chip (CoC) and Chip-on-Wafer (CoW) [2]. WoW is one of the most promising technologies as it uses metals for stacking wafers which helps in achieving an excellent electrical conductivity without compromising on the mechanical strength aspects. $\mathrm{Cu}-$ $\mathrm{Cu}$ WoW bonding is the preferred choice because of its high electromigration resistance [3]. The key challenges involved in carrying out $\mathrm{Cu}-\mathrm{Cu}$ WoW bonding include prevention of oxidation of $\mathrm{Cu}$ and protection of the same from surface contamination. Blanket $\mathrm{Cu}-\mathrm{Cu}$ bonding has been demonstrated using thermo-compression bonding by concurrent application of heat and pressure $[4,5]$. The bonding mechanism is based on inter-diffusion of $\mathrm{Cu}$ atoms and grain growth across the bonding interface. Pure copper is easily reactive with ambient oxygen and hence it gets oxidize. Oxide layer then acts as barrier for the inter-diffusion of $\mathrm{Cu}$ atoms. Hence for enhancing the diffusion process the oxide layer has to be removed prior to bonding. Surface roughness is also a critical factor in WoW blanket bonding. Contaminates present on surface really hampers the blanket bonding and also rough surface requires high temperature and pressure. An increase in surface roughness reduces the bonding strength as well as formation of voids due to uneven contact between two surfaces during bonding. This necessitates an ultra-smooth surface of sputter $\mathrm{Cu}$ thin films prior to bonding.

Researchers have reported several ways to protect the $\mathrm{Cu}$ surface from oxidation and contaminants. Kim et al. proposed a method is to clean the $\mathrm{Cu}$ surface prior to bonding and bond at ultra-high vacuum [6] but it is more time consuming and less manufacturing worthy. Tan et al. proposed a low temperature $\mathrm{Cu}-\mathrm{Cu}$ thermo-compression bonding $\left(\sim 250^{\circ} \mathrm{C}\right.$ and 2.5 bar) with temporary passivation of $\mathrm{Cu}$ surface by Self Assembled Monolayer (SAM) of alkane thiol [7, 8]. SAM is used to passivate the $\mathrm{Cu}$ surface immediately after sputtered $\mathrm{Cu}$ samples prior to bonding. SAM treatment on $\mathrm{Cu}$ surface not only provides protection from oxidation but also enhances the bonding quality. Though longer alkane thiol chains provide high degree of protection from oxidation but desorption of the same needs higher temperature [9]. Recently Y.P.Huang et al., proposed a novel bonding method using $\mathrm{Ti}$ as the passivation layer on $\mathrm{Cu}$ surface at $180^{\circ} \mathrm{C}$ temperature and $1.91 \mathrm{MPa}$ (19.1 bar) pressure [10]. However bonding pressure is too high to adopt in real CMOS applications. $\mathrm{Cu}$ is known to have a lower activation energy at the surface and a smaller atomic volume $\left(72 \mathrm{bohr}^{3}\right)$ than $\mathrm{Ti}$ vacancy volume $\left(75.48\right.$ bohr $\left.^{3}\right)[11,12]$. Hence, $\mathrm{Cu}$ has a tendency to diffuse towards the bonding interface through $\mathrm{Ti}$ vacancies, thereby 
reducing the effective surface roughness. If two wafers having $\mathrm{Cu}$ protected by ultra-thin $\mathrm{Ti}$ are brought together, the unoxidized copper diffuses through $\mathrm{Ti}$ towards the interface resulting in a $\mathrm{Cu}-\mathrm{Cu}$ bonding. The thickness of $\mathrm{Ti}$ plays a critical role in the whole process. Though thicker Ti films reduces surface roughness, it may decrease the diffusivity of copper in $\mathrm{Ti}$ thereby necessitating increased pressure conditions to achieve the bonding. On the other hand, very thin film may not able to neither passivate copper nor decrease surface roughness. An optimum Ti thickness needs to be chosen that would reduce the surface roughness and at the same time, allows $\mathrm{Cu}$ to diffuse through $\mathrm{Ti}$ and reach the interface.

In this work, a CMOS compatible WoW Cu-Cu thermocompression bonding method with $\mathrm{Ti}$ passivation was achieved at low temperature $\left(175^{\circ}\right)$ and low pressure $(2.5$ bar). Fig. 1 depicts the schematic of bonding protocol.

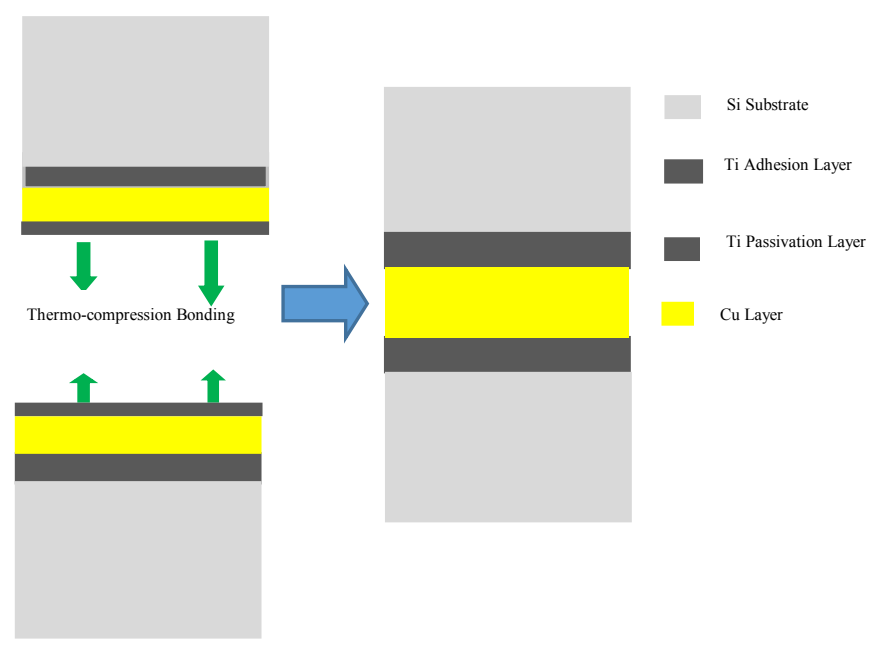

Figure 1. Schematic of CMOS compatible Bonding methodology

\section{Experimental}

In this whole experimental work we have utilized 4 inch Ptype $\langle 100\rangle$ Silicon wafers. The process flow can be categorized into three different parts Viz., (a) Cleaning of wafers (b) Thin film deposition (c) Bonding mechanism

\subsection{Cleaning of Wafers}

Standard RCA was used to clean the wafers. RCA-1 (Standard Clean-1), RCA-2 (Standard Clean-2) followed by piranha was used to clean both the wafers. This is to doubly ensure a complete remove of contamination prior to thin film deposition.

\subsection{Thin Film Deposition}

Thin film deposited on both the cleaned wafers in the following order $25 \mathrm{~nm}$ of Ti film, $250 \mathrm{~nm}$ of $\mathrm{Cu}$ film, followed by passivation layer of ultra-thin Ti using AJA multi-source sputtering system, USA in a base vacuum of $3 \times$ $10^{-2}$ torr at room temperature. The initial $\mathrm{Ti}$ layer below copper acts as adhesive layer between $\mathrm{Si}$ and $\mathrm{Cu}$. The role of passivation $\mathrm{Ti}$ layer is to protect the $\mathrm{Cu}$ surface from oxidation and contamination. In order to figure out the right thickness of ultra-thin Ti passivating layer, several pairs of $\mathrm{Cu}$ wafers were deposited with same conditions as mentioned above except for the passivation thickness which is varied for optimization purposes.

\subsection{Bonding Mechanism}

The bonding process was carried out in AML Alignment Wafer Bonder (AML-AWB, UK) machine by keeping both the metal film deposited wafers face to face on the wafer chuck. Vacuum pump was turned on to create vacuum up to $3 \times 10^{-3}$ Torr followed by 3 to 4 cycles of nitrogen purge in to the chamber. This ensures the removal of contamination and oxygen present in the chamber. After Nitrogen treatment, vacuum was created up to $5 \times 10^{-5}$ torr. Then the wafers were heated at a ramp rate of $46^{\circ} \mathrm{C} / \mathrm{min}$. Once the temperature reached up to $175^{\circ} \mathrm{C}, 2.5$ bar pressure was applied for the desired bonding duration in vacuum chamber. Then bonded wafers were allowed to cool down to room temperature which typically takes $2 \mathrm{hrs}$. Various characterization techniques were carried out to study the effect of Ti thickness on the quality bonding.

\section{Results and Discussion}

\subsection{Results of the Ti passivation surface prior to bonding}

\subsubsection{Surface Roughness}

Surface roughness is critical factor in WoW $\mathrm{Cu}-\mathrm{Cu}$ blanket bonding. An increase in surface roughness reduces the bonding strength since the bonding quality depends on the surface quality. Rough surface requires higher bonding pressure could disturb device reliability. In this endeavor, $\mathrm{Ti}$ thickness was optimized by varying thickness of $\mathrm{Ti}$ and performing AFM studies to study the effect of surface roughness prior to bonding. AFM (Bruker Icon ScanAsyst) was used to investigate the surface roughness of the $\mathrm{Ti}$ passivation layer. In the whole AFM analysis imaging was done in tapping mode for $1 \mu \mathrm{m}^{2}$ scan area. The tapping mode 3D AFM image of sputtered $\mathrm{Cu}$ film is shown in Fig. 2. The RMS roughness of the film is $2.1 \mathrm{~nm}$. The brighter areas in the image clearly indicate the presence of native oxide on $\mathrm{Cu}$. Fig. 3 shows the variation of RMS roughness of $\mathrm{Cu} / \mathrm{Ti}$ surface for various thicknesses of passivation. The RMS roughness obtained of ultra-thin $\mathrm{Ti}(3 \mathrm{~nm})$ coated $\mathrm{Cu}$ sample was observed $0.414 \mathrm{~nm}$ which is the lowest roughness that could be achieved and is very less compared to roughness of as deposited $\mathrm{Cu}$ without $\mathrm{Ti}$ passivation. Though the roughness obtained for higher Ti thicknesses $(0.45 \mathrm{~nm}, 0.463 \mathrm{~nm}, 0.501$ $\mathrm{nm}$ for $5 \mathrm{~nm}, 7 \mathrm{~nm}$ and $9 \mathrm{~nm}$ Ti passivation layer thicknesses respectively), the corresponding wafers could not be bonded properly at lower pressure because of higher RMS roughness as compared to $3 \mathrm{~nm}$ passivation layer. This can be attributed to the hindrance of $\mathrm{Cu}$ diffusivity through $\mathrm{Ti}$ to the bonding interface owing to the higher thickness of Ti. Furthermore, for $2 \mathrm{~nm}$ passivation layer have higher RMS roughness $(0.469$ $\mathrm{nm})$ as compared to $3 \mathrm{~nm}$ passivation layer on above $\mathrm{Cu}$ film. 
This can be attributed to insufficient thickness of passivation layer. This would have allowed the formation of native copper oxide which is responsible for increase in the roughness. Hence it can be inferred from the above investigation that 3 $\mathrm{nm} \mathrm{Ti}$ on $\mathrm{Cu}$ thin film reduces the surface roughness of $\mathrm{Cu}$ and it may result in lower the temperature and pressure of bonding.

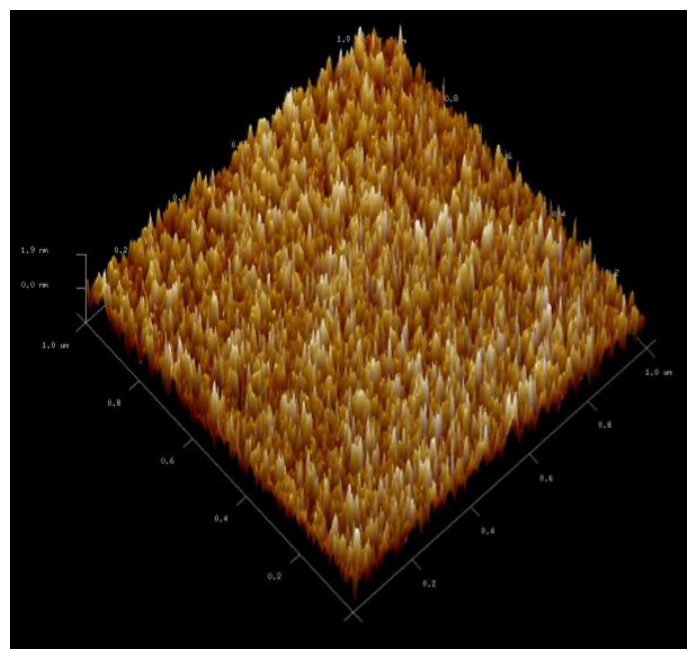

Figure 2. 3D imaging of Pure $\mathrm{Cu}$ extracted from AFM.

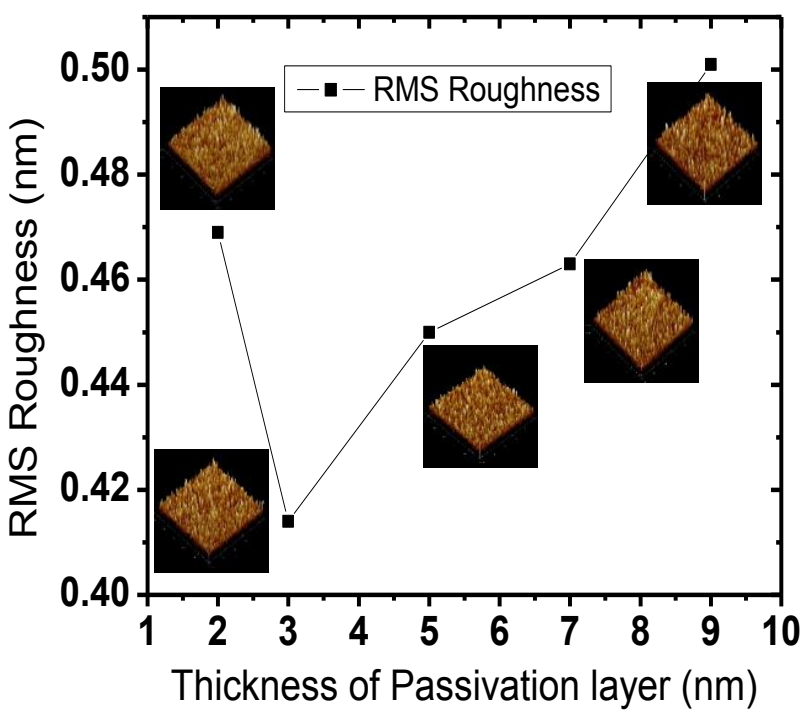

Figure 3. Comparison graph extracted from AFM Study. Roughness is minimal for Ti thickness of $3 \mathrm{~nm}$.

\subsubsection{Chemical Analysis}

After figuring out the Ti thickness required to minimize the surface roughness, it is imperative to find out the thickness of $\mathrm{Ti}$ that is essential to prevent $\mathrm{Cu}$ from oxidation. For this purpose, the chemical constitution of the $\mathrm{Ti}$ passivated $\mathrm{Cu}$ films were analyzed by Energy Dispersive X-ray Spectroscopy (EDX) for various values of Ti thickness. Though EDX is not a surface analysis, it gives a good insight about the individual elements and their weight percentage. Specifically the percentage of oxygen present in the overall composition is an indicative of the amount of oxidation that has happened on the surface. For Ti thickness of $3 \mathrm{~nm}$ the overall oxygen content is minimal as compared to the other thicknesses (Fig. 4). The high amount of oxygen content for other thickness may also be attributed to the oxidation $\mathrm{Ti}$ since $\mathrm{Ti}$ is known to form native oxides as well. However that is not of a major concern to us as the primary target is passivated $\mathrm{Cu}$ from getting oxidized. The minimal oxygen weight at a Ti thickness of $3 \mathrm{~nm}$ is indicative of minimal oxidation of $\mathrm{Ti}$ and successful passivation of $\mathrm{Cu}$. Thus the EDX results correlates well with the findings of AFM studies.

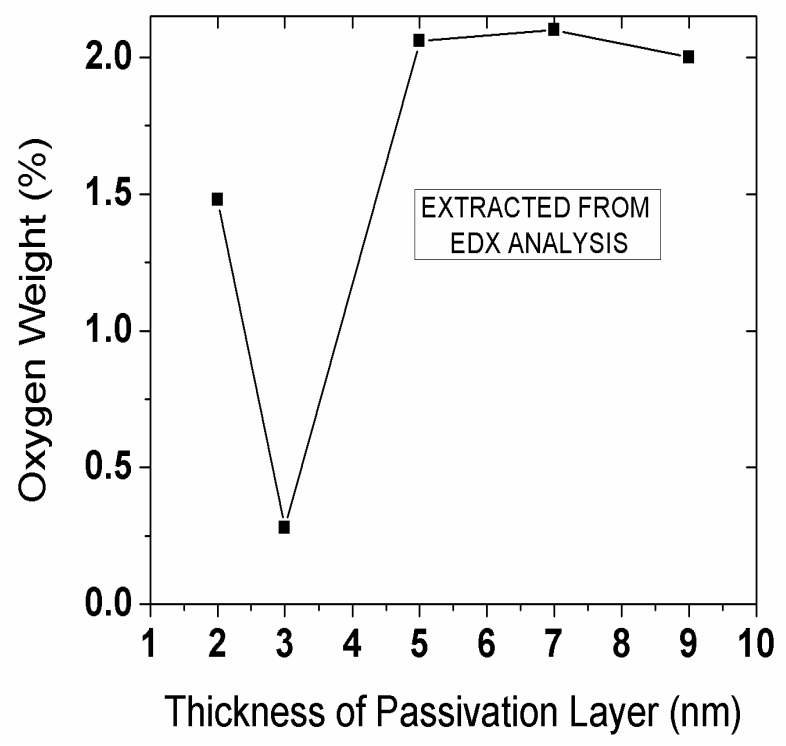

Figure 4. Oxygen Weight (\%) extracted from EDX. Oxygen content is minimal for Ti thickness of $3 \mathrm{~nm}$.

\subsection{Results of the $\mathrm{Cu}-\mathrm{Cu}$ interface after wafer bonding}

\subsubsection{Interface Qualification}

Uneven voids in the interface degrade the bonding quality as well as bond strength. In order to identify the manifestation of voids, C- Mode Scanning Acoustic Microscope (C-SAM, Sonoscan, UK) observation was carried out. This is one of the best non-destructive qualitative technique which is regularly used for analyzing the interface of the bonded sample. It is a very high frequency pulse-echo ultrasonic microscope which provides images by mechanically scanning a transducer over a sample. A highly focused beam of ultrasound, generated by an acoustic lens, is focused onto the bonded interface by a coupling medium which typically is water. The transducer (whose frequency of operation is $100 \mathrm{MHz}$ ) plays a vital role of both sending and receiving of the pulses. Pulses focused at the interface generate a corresponding echo due to the interfacial properties [13]. The intensity of the reflected echo 
is monitored and is converted into an image. A dark pixel in the image indicates a high intensity of the echo. Thus if the interface is defect free, the intensity of the echoes reflected are high. On the contrary, a defect would generate an echo of low intensity which gets mapped as a bright pixel in the generated acoustic image. The inherent dark image suggests a very good bonding with Ti passivation with optimal thickness of $3 \mathrm{~nm}$ (Fig. 5). Very Sparse voids appearing as a bright spots may be attributed to the presence of dust/contamination on the wafer just prior to bonding.

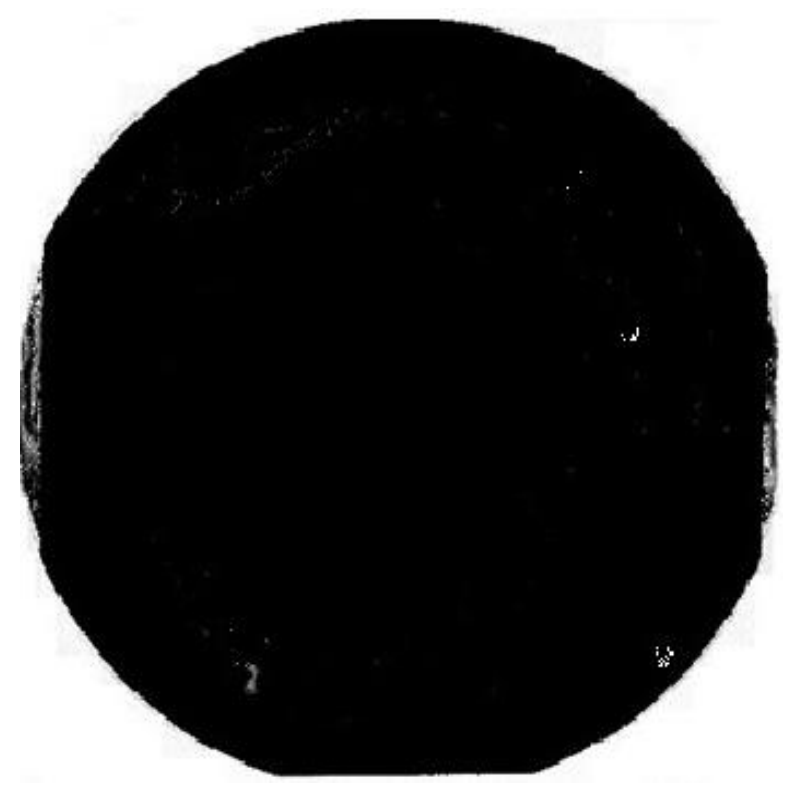

Figure 5. C-SAM imaging for bond qualification ( $\mathrm{Ti}$ Passivation Thickness $=3 \mathrm{~nm}$ ).

The C-SAM image for a Ti thickness of $4 \mathrm{~nm}$ (Fig. 6) shows a predominant dark area and sparse bright area. Here the bright area cannot be attributed to the dust particles and may be attributed to a relatively weak bonding at the interface.

The C-SAM image for Ti thickness of $5 \mathrm{~nm}$ (Fig.7) shows a higher amount of bright area indicating that interface quality is still poorer. Beyond this thickness, bonding between two wafers was not observed which emphasizes our motivation to figure out the right thickness of passivation required for achieving a good quality bonding. The C-SAM image for $\mathrm{Ti}$ thickness of $2 \mathrm{~nm}$ (not shown), also had the same features as that of higher thicknesses indicating that the quality of the bonding is inferior compared to the case wherein the passivation Ti thickness is $3 \mathrm{~nm}$. The reason for poor bonding at higher thicknesses may be attributed to the inadequate $\mathrm{Cu}$ at the interface whereas at lower thickness of $2 \mathrm{~nm}$, the poor bonding may be attributed to inadequate passivation which would have not prevented the formation of native oxide on $\mathrm{Cu}$ surface. These inferences are further corroborated with thorough bond strength analysis carried out using razor test and microtester.

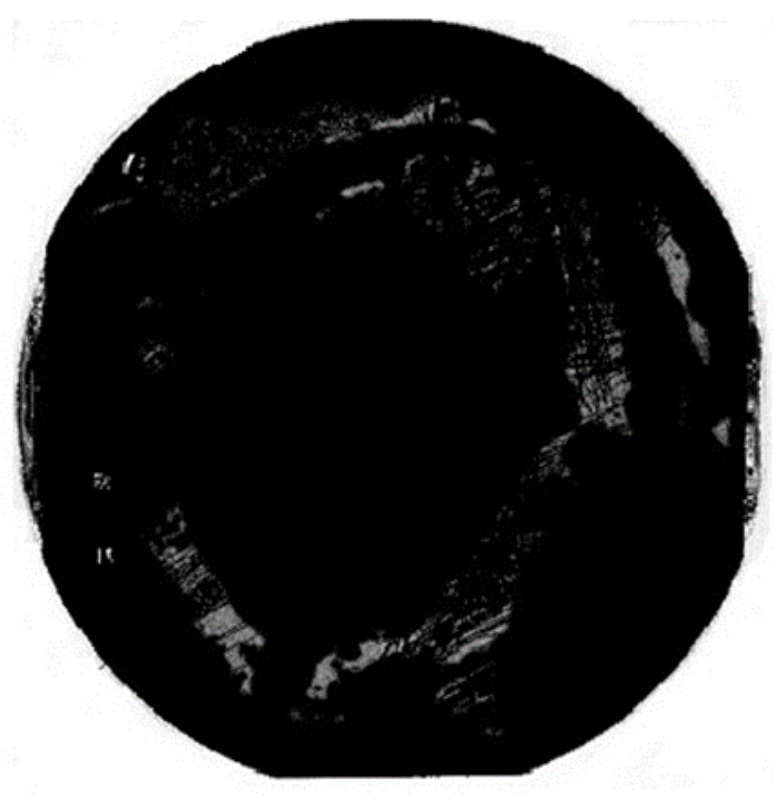

Figure 6. C-SAM imaging for bond qualification ( $\mathrm{Ti}$ Passivation Thickness $=4 \mathrm{~nm}$ ).

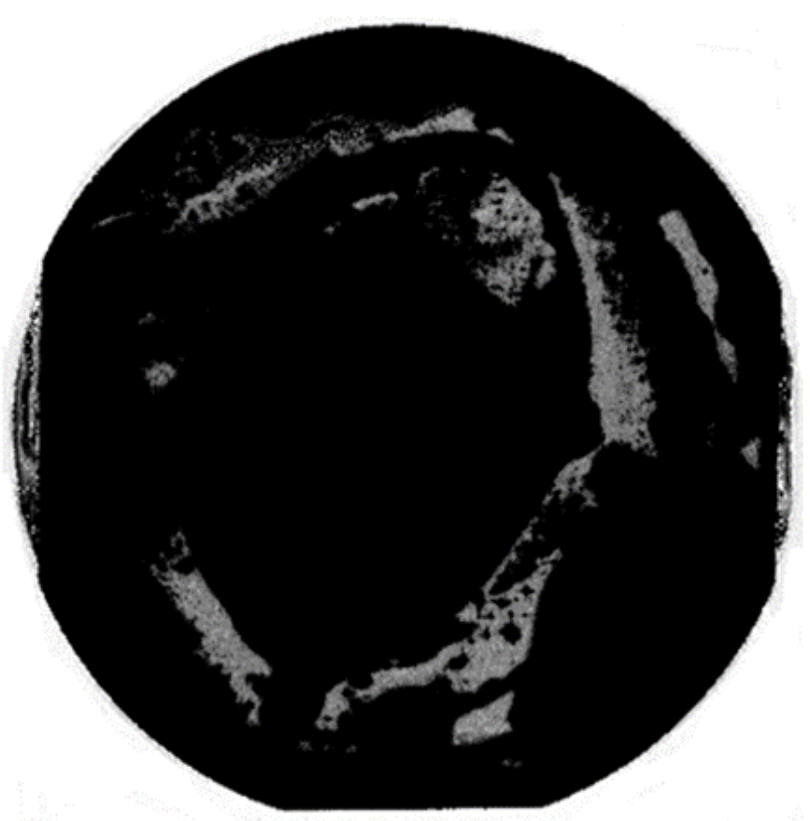

Figure 7. C-SAM imaging for bond qualification ( $\mathrm{Ti}$ Passivation Thickness $=5 \mathrm{~nm}$ ).

\subsubsection{Bond Strength Analysis of the Bonded Sample}

Initially the bond strength characterization was carried out using a simple razor blade test. A high quality bonding would not allow the razor to penetrate at the interface. Partial penetration of razor blade was observed when the thickness of the Ti film is more than or equal to $4 \mathrm{~nm}$. The degree of 
penetration increased with the Ti thickness suggesting that higher Ti thickness is not suitable for achieving a good quality bonding. However at an optimal thickness of $3 \mathrm{~nm}$, the razor blade test indicated that the quality of bonding is good and no penetration at the interface was observed. This was further corroborated by carrying of bond strength analysis. The bond strength of $\mathrm{Cu}-\mathrm{Cu}$ as measured by Instron Microtester measurement system as shown in Fig. 8 is found to be 190 $\mathrm{MPa}$ which compares very well with the reported literatures.

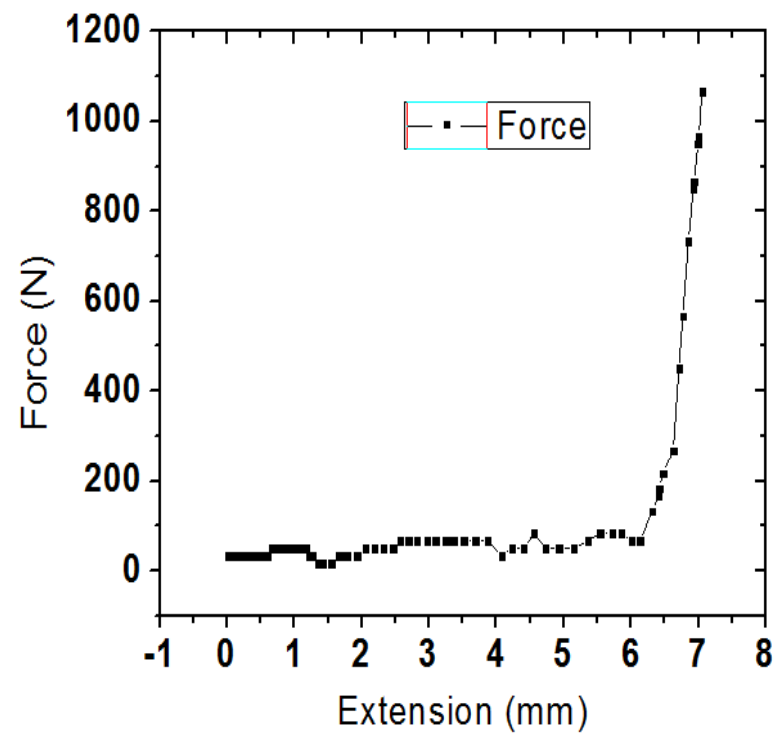

Figure 8. Bond Strength Qualification

\subsubsection{Microstructure Imaging}

Cross-sectional imaging analysis is a qualitative technique which is used to assess the quality of bonded interface Uneven voids at the interface reduces the quality of bonding as well bond strength. The bonded samples were cut into $1 \mathrm{~cm} \times 1 \mathrm{~cm}$ pieces using a diamond scriber. The wafer bonding was intact even after the scribing which indicates a good quality interface. In order to identify the presence of intermediate layers and voids, Field Emission-Single Electron Microscopy (FE-SEM, ZEISS) was carried out. Fig. 9 shows the cross section FE-SEM image of the bonded layer at medium magnification of $100 \mathrm{~K} \mathrm{X}$. No interface was observed between two $\mathrm{Cu}$ layers at this magnification or even at higher magnification $833.97 \mathrm{~K} \mathrm{X}$ (Fig. 10). The absence of interface as well as the absence of layered structure is a very good indication that layers have interdiffused among each other and have formed a single layer instead of four individual layers.

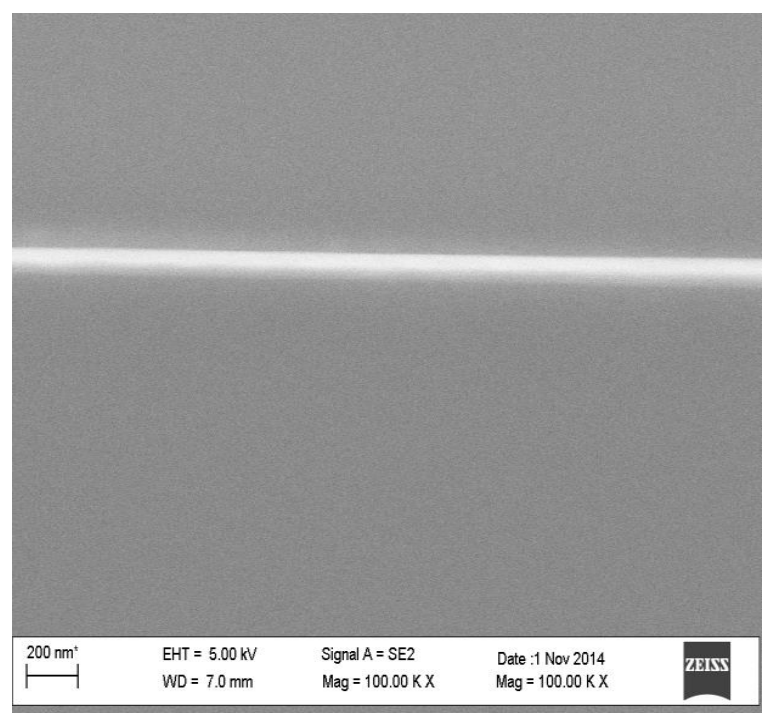

Figure 9. Medium resolution micrograph using FE-SEM

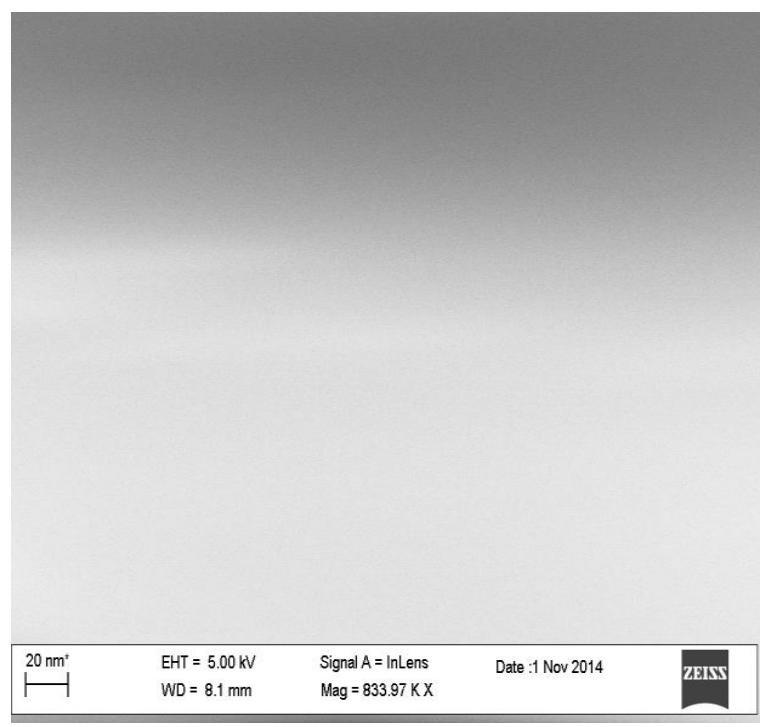

Figure 10. High resolution micrograph using FE-SEM

\section{Conclusions}

Wafer on Wafer $\mathrm{Cu}-\mathrm{Cu}$ thermos-compression bonding was successfully demonstrated at low temperature $\left(175^{\circ} \mathrm{C}\right)$ and low pressure ( 2.5 bar) using optimally chosen ultra- thin layer of $\mathrm{Ti}(3 \mathrm{~nm})$ as a passivation layer. Various characterization techniques were meticulously carried out to optimize the $\mathrm{Ti}$ thickness. This systematic study has resulted in bonding conditions which are physically viable for 3D IC Integration applications. Our optimized result yielded a very good bond strength of $\mathrm{Cu}-\mathrm{Cu}$ as corroborated by Instron Microtester measurements, C-SAM and FE-SEM images. Specifically the 
absence of interface in the cross sectional FE-SEM images indicates a very high quality bonding.

\section{Acknowledgments}

Authors are grateful with funding support from the Department of Electronics \& Information Technology (DeitY), Govt. of India who have funded us this project. Authors are also grateful for the support that they received from INUP program that is being carried out by Centre for Nanoscience and Engineering (CeNSE), Indian Institute of Science, Bangalore.

\section{References}

1. C.S. Tan, R. J. Gutmann, and L. R. Reif, Wafer level 3-D ICs process technology, Springer Science \& Business Media, 2009, pp. 3.

2. C.S. Tan, R. J. Gutmann, and L.R. Reif, Overview of Wafer-Level 3D ICs, Springer, US, 2008, pp. 1-11.

3. D. Save, F. Braud, J. Torres, F. Binder, C. Müller, J. O. Weidner, and W. Hasse. "Electromigration resistance of copper interconnects," Microelectronic engineering 33, vol. 1, 1997, pp. 75-84.

4. K.N.Chen, C.S.Tan, A.Fan, and L. R.Reif, "Morphology and bond strength of copper wafer bonding," Electrochemical and Solid-State Letters 7, vol. 1, 2004, pp. G14-G16.

5. E-J.Jang, S.Hyun, H-J.Lee, and Y-B.Park, "Effect of wet pretreatment on interfacial adhesion energy of $\mathrm{Cu}-\mathrm{Cu}$ thermocompression bond for 3D IC packages," Journal of electronic materials 38, vol. 12, 2009, pp. 2449-2454.

6. T. H. Kim, M. M. R. Howlader, T. Itoh, and T. Suga, "Room temperature $\mathrm{Cu}-\mathrm{Cu}$ direct bonding using surface activated bonding method," Journal of Vacuum Science \& Technology A 21,vol. 2, 2003, pp. 449-453.

7. C. S. Tan, D. F. Lim, S. G. Singh, S. K. Goulet, and M. Bergkvist, " $\mathrm{Cu}-\mathrm{Cu}$ diffusion bonding enhancement at low temperature by surface passivation using self-assembled monolayer of alkane-thiol," Applied Physics Letters 95, vol. 19, 2009, pp. 192108.

8. C.S.Tan, D.F.Lim, X.F.Ang, J.Wei, and K.C.Leong, "Low temperature $\mathrm{Cu}-\mathrm{Cu}$ thermo-compression bonding with temporary passivation of self-assembled monolayer and its bond strength enhancement." Microelectronics Reliability 52, vol. 2, 2012, pp. 321-324.

9. D. F. Lim, J. Wei, K. C. Leong, and C. S. Tan, "Cu passivation for enhanced low temperature $\left(\leqslant 300^{\circ} \mathrm{C}\right)$ bonding in 3D integration." Microelectronic Engineering 106, 2013, pp. 144-148.

10. Y.P. Huang, Y. S. Chien, R.N.Tzeng, M.S.Shy, T.H.Lin, K.H.Chen, C.T.Chiu, J.C.Chiou, C.T.Chuang, W.Hwang, H.M.Tong, and K.N.Chen, "Novel Cu-to- $\mathrm{Cu}$ Bonding With Ti Passivation at $180^{\circ}$ in 3-D Integration." Electron Device Letters, IEEE 34, vol. 12, 2013, pp. 1551-1553.

11. P. A. Korzhavyi, I. A. Abrikosov, B. Johansson, "Firstprinciples calculations of the vacancy formation energy in transition and noble metals." Physical Review B 59, vol. 18, 1999, pp. 11693.
12. E. S. Machlin, An introduction to aspects of thermodynamics and kinetics relevant to materials science, Elsevier, 2010, pp. 251.

13. Zuliang $\mathrm{Yu}$, and Siegfried Boseck. "Scanning acoustic microscopy and its applications to material characterization." Reviews of Modern Physics 67, vol. 4, 1995, pp. 863. 